\title{
Cell Intrinsic Roles of Apoptosis-Associated Speck-Like Protein in Regulating Innate and Adaptive Immune Responses
}

\author{
Hoda Hassan and Amal O. Amer \\ Department of Microbial Infection and Immunity, Center for Microbial Interface Biology, \\ and Department of Internal Medicine, The Ohio State University, Columbus, OH 43210, USA
}

Received 10 November 2011; Accepted 14 November 2011

Academic Editor: Marco Antonio Cassatella

The role of apoptosis-associated speck-Like protein (ASC) in the assembly of the inflammasome complex within macrophages has been elucidated in several studies. In this particular role, ASC functions as an adaptor protein by linking nod-like receptors (NLRs) and procaspase-1, thereby leading to the activation of caspase- 1 to cleave inflammatory cytokines IL-1 $\beta$ and IL-18 and inducing pyroptosis. It has been noted that ASC maintains inflammasome-independent roles, including but not limited to controlling the expression of Dock2 and mitogen-activated protein kinases (MAPK/ERK2) and regulating the NF- $\kappa \mathrm{B}$ pathway. This paper will emphasize the major roles of ASC during pathogen infection, the mechanisms by which it mediates inflammation, and discuss its more recently discovered functions.

KEYWORDS: apoptosis-associated speck-like protein (ASC), caspase-1, inflammasome, nod-like receptors (NLRs), pyroptosis 


\section{INTRODUCTION}

The apoptosis-associated speck-like protein containing a caspase recruitment domain ASC is an adaptor molecule consisting of an $\mathrm{N}$-terminal pyrin domain (PYD) and a $\mathrm{C}$-terminal caspase recruitment domain (CARD; [1-3]). Both domains are utilized during the establishment of the inflammasome, a multiprotein complex that plays a key role in the innate immune response to infection [3-5]. While ASC has primarily been studied within the context of the inflammasome, it appears to have a variety of uncharacterized roles.

The process of inflammatory response by the inflammasome begins with recognition of damageassociated molecular patterns (DAMPs) by nucleotide-binding domain, leucine-rich repeat containing proteins (NLR; [6-8]). This diverse group of proteins includes CARD-containing NLRs, Naip proteins, and NLRs containing pyrin domains [9]. The recognition of DAMPs initiates the assembly of the inflammasome, a cytosolic protein complex composed of NLRs, procaspase-1, and ASC [10, 11].

The proteins Nlrp3, Nlrc4, Nlrp1, and Aim2 allow the formation of inflammasome complexes that have been described in detail while a potentially fifth inflammasome, pyrin, is being investigated $[5,12,13]$. Recent studies have shown that most inflammasome complexes require ASC to assemble [9, 14]. This is because most NLRs lack a CARD domain which is necessary for the interaction with caspase-1. Nlrc4 and Nlrp1 contain a CARD domain which was suggested to interact with caspase-1. Accordingly, the Nlrc4mediated process of pyroptosis does not necessitate the inclusion of ASC.

\section{THE ROLE OF ASC WITHIN THE NLRC4 INFLAMMASOME}

The much-studied protein Nlrc4 provides a comprehensive example of how such an assembly forms in response to Legionella $(L$.) pneumophila infection. Following the detection of bacterial flagellin in murine macrophages, Nlrc4 interacts with ASC through the pyrin domain [8, 10]. Afterwards, ASC recruits caspase- 1 through its CARD domain. Then, the protease cleaves inflammatory cytokines IL- $1 \beta$, and IL18 (Figure 1). The secretion of caspase-1, IL-1 $\beta$ and IL-18 promotes pore formation and a specialized form of cell death termed pyroptosis [15]. The aforementioned assembly of the Nlrc4 inflammasome has received considerable attention due to the controversy surrounding the participation of ASC. Multiple studies have clarified that ASC contributes, at least in part, to the activation of caspase- 1 independently of Nlrc4 $[3,9,10]$. Recent studies by Abdelaziz et al. $[3,10]$ have further delineated two pathways and have described how ASC can independently contribute to inflammation and caspase-1 activation (Figure 1). ASC has been found to control L. pneumophila infection in murine macrophages that lack caspase-1 [3]. This was confirmed by downregulating ASC in wild-type (WT) and caspase-1 knockout macrophages and infecting with L. pneumophila. WT macrophages are restrictive to Legionella infection whereas caspase$1^{-/-}$macrophages are permissive. In the caspase- $1^{-/-}$cells, the depletion of ASC allowed for greater replication of the bacterium whereas there was no effect in the WT macrophages [3].

While ASC is certainly essential in activating caspase-1 during L. pneumophila infection, treating murine macrophages lacking ASC with L. pneumophila still permitted the cleavage of procaspase-1, albeit at a smaller fraction than WT macrophages [3]. However, the absence of ASC diminished the release of IL-1 $\beta$ from macrophages, suggesting that not all inflammatory responses are independent of ASC [3]. Furthermore, caspase-1 was cleaved in $\mathrm{ASC}^{-/-}$macrophages only in the presence of flagellin [3]. This result suggests that flagellin is required for caspase-1 activation. The authors examined whether or not $L$. pneumophila lacking flagellin (Fla) was able to grow in the presence or absence of ASC. They found that $\mathrm{ASC}^{-/-}$macrophages allowed for significantly more bacterial growth in comparison to WT macrophages. This discovery suggests that ASC maintains an alternative pathway to control L. pneumophila infection [3].

Specifically, it was found that caspase-3, which is involved in apoptosis, was activated in WT macrophages but not in $\mathrm{ASC}^{-/-}$macrophages $[3,16]$. This prompted the conclusion that ASC is involved in the activation of caspase-3, which mediates apoptosis during later stages of infection (Figure 1) [3].

Studies have also shown that high levels of extracellular potassium in macrophages inhibit the activation of caspase-1 in those inflammasomes that require ASC [9]. This is because high potassium levels 


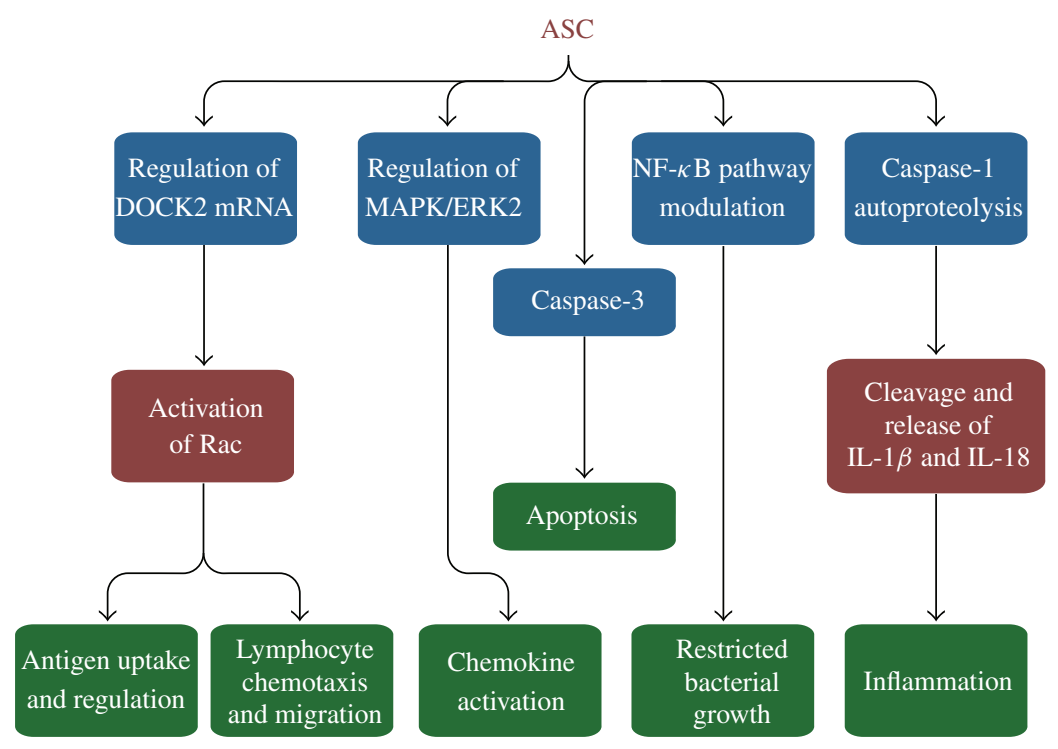

FIGURE 1: ASC Effector Mechanisms. ASC is involved in innate and adaptive immune responses. ASC modulates inflammation, cell survival, cell death, chemokine activation, lymphocyte chemotaxis, and antigen uptake and presentation. Other functions of ASC have also been revealed that are independent of an inflammasome complex, including regulation of the NF- $\kappa$ B pathway and MAPK activation as well as controlling the stability of Dock2 mRNA.

interfere with the oligomerization of ASC, a crucial step in activating caspase-1 [9]. The authors noted that the Nlrc4 inflammasome was unaffected by potassium levels, further emphasizing the distinct differences between inflammasome complexes.

Human monocytes are permissive to L. pneumophila infection due to the lack of caspase- 1 activation. In this study, Abdelaziz et al. [10] revealed that L. pneumophila infection significantly decreased the level of ASC, along with decreasing the levels of caspase-1, NLRC4, and IL-1 $\beta$ in human macrophages [10]. Thus, L. pneumophila modulated the levels of ASC and other important inflammatory components to avoid caspase- 1 activation and permit its own growth.

To determine the importance of human ASC in activating caspase-1, ASC was ectopically expressed in human monocytes before infection with L. pneumophila [10]. This resulted in the cleavage of procaspase1. Thus, expression of ectopic ASC is capable of restoring caspase-1 activation in human monocytes.

Previous reports have demonstrated that ASC exists diffusely throughout the cell, but most have relied on overexpressing ASC to arrive at this conclusion [8]. On the other hand, it was found that endogenous ASC localized to the nucleus in resting human macrophages, forming characteristic aggregates [8]. Immunofluorescence studies showed that ASC in fact relocalized to the cytosol during E. coli infection, thus suggesting that this translocation is in response to inflammatory activation of macrophages [8]. Additional studies revealed that impeding the nuclear export of ASC prevented the release of IL-1 $\beta$ [8]. Therefore, the mechanism in which ASC localizes in the cytosol due to pathogen infection is an important modulator of cytokine release.

\section{MODULATING THE NF- $\kappa$ B PATHWAY: AN INFLAMMASOME-INDEPENDENT ROLE}

ASC can modulate the growth of L. pneumophila through manipulation of the nuclear factor $\kappa \mathrm{B}(\mathrm{NF}-\kappa \mathrm{B})$ pathway [17]. L. pneumophila is thought to activate NF- $\kappa \mathrm{B}$ through two phases, one is a transient process dependent on TLR5, Myd88, and flagellin while the other is more long lasting and functions independently of the aforementioned proteins [18]. Activation of the NF- $\kappa$ B pathway is associated with increased 
L. pneumophila growth because it extends the survival of the host cell. Abdelaziz et al. [3, 10] have revealed that both murine and human macrophages utilize ASC in hindering NF- $\kappa$ B pathway activation and restricting bacterial survival (Figure 1). Caspase- $1^{-/-}$murine macrophages were depleted of ASC and then infected with $L$. pneumophila $[3,10]$. They permitted greater NF- $\kappa \mathrm{B}$ activation and enhanced L. pneumophila growth [3, 10]. In human monocytic cell line THP-1 cells, the exogenous expression of ASC impeded the activation of the NF- $\kappa \mathrm{B}$ pathway and limited the growth of L. pneumophila [3, 10]. Therefore, ASC can control the infection of L. pneumophila in an inflammasome and caspase-1-independent manner.

\section{DOCK2 AND MAPK/ERK2: NOVEL TARGETS UNDER ASC CONTROL}

Increased interest in ASC and its role in innate immunity has directed attention to clarifying its other functions outside of the realm of the inflammasome. The guanine nucleotide-exchange factor Dock2 mediates actin polymerization through regulating the GTPase Rac [5, 19]. Dock2-mediated Rac activation mediates different types of immune response, such as antigen uptake and presentation and lymphocyte chemotaxis and migration [5]. Comparison of antigen uptake and presentation in murine dendritic cells (DCs) lacking ASC with WT macrophages demonstrated that DCs not expressing ASC failed to elicit proper T-cell response [5]. Further studies using $\mathrm{ASC}^{-/-}$bone marrow-derived cells (BMDCs) proved that these cells were defective in phagocytosis [5]. Using $\mathrm{ASC}^{-/-} \mathrm{T}$ and $\mathrm{B}$ cells, Ippagunta et al. [5] demonstrated that the omission of ASC greatly impaired the natural migratory ability of lymphocytes. Both processes are dependent on functional Rac activation, which was found to be invariably linked to the presence or absence of ASC within the cell. Interestingly, the authors relayed the possibility that nuclear but not cytosolic ASC was responsible for regulating Dock2 mRNA (Figure 1).

Additionally, ASC has been identified as a regulator of MAPK/ERK2 activity in murine and THP-1 macrophages [11]. MAPKs are a family of signaling proteins that control a diverse group of functions, including ones pertaining to innate immune response such as chemokine activation [11]. DUSP10, which negatively regulates MAPK activity through dephosphorylation, works in tandem with ASC. ASC regulates MAPK activity through phosphorylation independently of caspase-1 and describes a novel target of ASC.

ASC has been associated with proper granuloma formation and host defense during Mycobacterium tuberculosis infection and activating caspases 2 and 3 during regulation of a p53-bax mitochondrial pathway [5]. Additionally, the Pycard/TMS1 (target of methylation) gene that encodes for ASC has been found to suppress the proliferation of certain tumors by inducing apoptosis [20].

\section{CONCLUSION}

The adaptor molecule ASC has multiple roles in innate immune response. The most well known of these roles is its mediation of the assembly of the inflammasome by linking NLRs with procaspase-1. Detailed analyses of experimentation with murine and human macrophages in the context of L. pneumophila infection have revealed the unique roles of ASC in mediating inflammation. Other functions of ASC have also been revealed that are independent of an inflammasome complex, including regulation of the NF- $\kappa \mathrm{B}$ pathway and MAPK activation as well as controlling the stability of Dock2 mRNA. Future studies may well uncover additional roles of ASC within the cell.

\section{ACKNOWLEDGMENT}

The authors thank Dr. Tim Eubank for Figure 1. 


\section{REFERENCES}

[1] J. Masumoto, S. Taniguchi, K. Ayukawa et al., "ASC, a novel 22-kDa protein, aggregates during apoptosis of human promyelocytic leukemia HL-60 cells,” Journal of Biological Chemistry, vol. 274, no. 48, pp. 33835-33838, 1999.

[2] K. Matsushita, M. Takeoka, J. Sagara et al., "A splice variant of ASC regulates IL-1beta release and aggregates differently from intact ASC," Mediators of Inflammation, vol. 2009, Article ID 287387, p. 6, 2009.

[3] D. H. Abdelaziz, M. A. Gavrilin, A. Akhter et al., "Asc-dependent and independent mechanisms contribute to restriction of Legionella pneumophila infection in murine macrophages," Frontiers in Microbiology, vol. 2, p. 18, 2011.

[4] F. Martinon, K. Burns, and J. Tschopp, "The Inflammasome: a molecular platform triggering activation of inflammatory caspases and processing of proIL- $\beta$," Molecular Cell, vol. 10, no. 2, pp. 417-426, 2002.

[5] S. K. Ippagunta, R. K.S. Malireddi, P. J. Shaw et al., "The inflammasome adaptor ASC regulates the function of adaptive immune cells by controlling Dock2-mediated Rac activation and actin polymerization," Nature Immunology, vol. 12, no. 10, pp. 1010-1016, 2011.

[6] D. M. Underhill, A. Ozinsky, A. M. Hajjar et al., "The Toll-like receptor 2 is recruited to macrophage phagosomes and discriminates between pathogens," Nature, vol. 401, no. 6755, pp. 811-815, 1999.

[7] J. L. Poyet, S. M. Srinivasula, M. Tnani, M. Razmara, T. Fernandes-Alnemri, and E. S. Alnemri, "Identification of Ipaf, a human Caspase-1-activating protein related to Apaf-1," Journal of Biological Chemistry, vol. 276 , no. 30, pp. 28309-28313, 2001.

[8] N. B. Bryan, A. Dorfleutner, Y. Rojanasakul, and C. Stehlik, "Activation of inflammasomes requires intracellular redistribution of the apoptotic speck-like protein containing a caspase recruitment domain," Journal of Immunology, vol. 182, no. 5, pp. 3173-3182, 2009.

[9] C. L. Case, S. Shin, and C. R. Roy, "Asc and Ipaf inflammasomes direct distinct pathways for caspase-1 activation in response to Legionella pneumophila," Infection and Immunity, vol. 77, no. 5, pp. 1981-1991, 2009.

[10] D. H. Abdelaziz, M. A. Gavrilin, A. Akhter et al., "Apoptosis-associated speck-like protein (ASC) controls Legionella pneumophila infection in human monocytes," Journal of Biological Chemistry, vol. 286, no. 5, pp. 3203-3208, 2011.

[11] D. J. Taxman, E. A. Holley-Guthrie, M. T. Huang et al., "The NLR adaptor ASC/PYCARD regulates DUSP10, mitogen-activated protein kinase (MAPK), and chemokine induction independent of the inflammasome," Journal of Biological Chemistry, vol. 286, no. 22, pp. 19605-19616, 2011.

[12] A. Akhter, M. A. Gavrilin, L. Frantz et al., "Caspase-7 activation by the nlrc4/ipaf inflammasome restricts Legionella pneumophila infection," PLoS Pathogens, vol. 5, no. 4, Article ID e1000361, 2009.

[13] M. S. F. Pereira, G. G. Marques, J. E. DelLama et al., "The Nlrc4 inflammasome contributes to restriction of pulmonary infection by Flagellated legionella spp. that trigger pyroptosis," Frontiers in Microbiology, vol. 2, no. 33, pp. 1-6, 2011.

[14] A. Stutz, D. T. Golenbock, and E. Latz, "Inflammasomes: too big to miss," Journal of Clinical Investigation, vol. 119, no. 12, pp. 3502-3511, 2009.

[15] C. L. Case, S. Shin, and C. R. Roy, "Asc modulates the function of NLRC4 in response to infection of macrophages by Legionella pneumophila," mBio, vol. 2, no. 4, 2011.

[16] M. Molmeret, S. D. Zink, L. Han et al., "Activation of caspase-3 by the Dot/lcm virulence system is essential for arrested biogenesis of the Legionella-containing phagosome," Cellular Microbiology, vol. 6, no. 1, pp. 33-48, 2004.

[17] A. A. Khweek and A. O. Amer, "Replication of Legionella pneumophila in human cells: why are we Susceptible?" Frontiers in Microbiology, vol. 1, p. 133, 2010.

[18] V. P. Losick and R. R. Isberg, "NF- $\kappa$ B translocation prevents host cell death after low-dose challenge by Legionella pneumophila,” Journal of Experimental Medicine, vol. 203, no. 9, pp. 2177-2189, 2006.

[19] K. Gotoh, Y. Tanaka, A. Nishikimi et al., "Selective control of type I IFN induction by the Rac activator DOCK2 during TLR-mediated plasmacytoid dendritic cell activation," Journal of Experimental Medicine, vol. 207, no. 4, pp. 721-730, 2010.

[20] A. K. Siraj, A. R. Hussain, M. Al-Rasheed et al., "Demthylation of TMS1 gene sensitizes thyroid cells to tRAILinduced apoptosis," The Journal of Clinical Endocrinology \& Metabolism, vol. 96, no. 1, pp. E215-E224, 2011. 


\section{This article should be cited as follows:}

Hoda Hassan and Amal O. Amer, "Cell Intrinsic Roles of Apoptosis-Associated Speck-Like Protein in Regulating Innate and Adaptive Immune Responses," TheScientificWorldJOURNAL, vol. 11, pp. 24182423, 2011. 


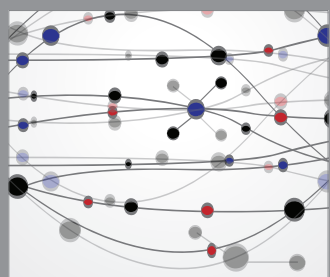

The Scientific World Journal
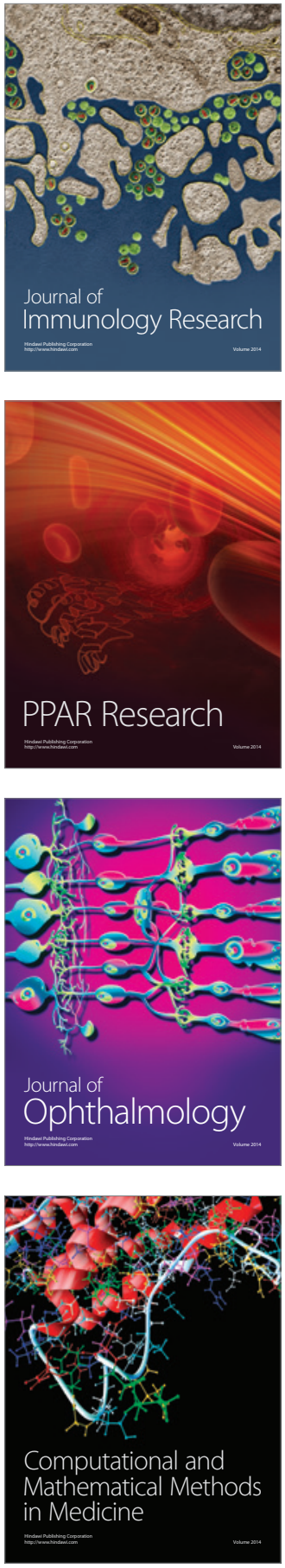

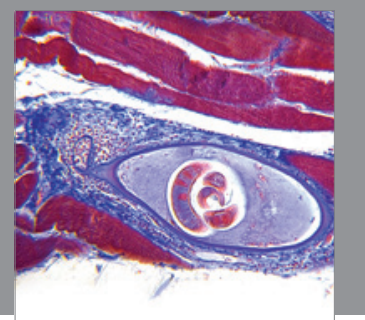

Gastroenterology

Research and Practice
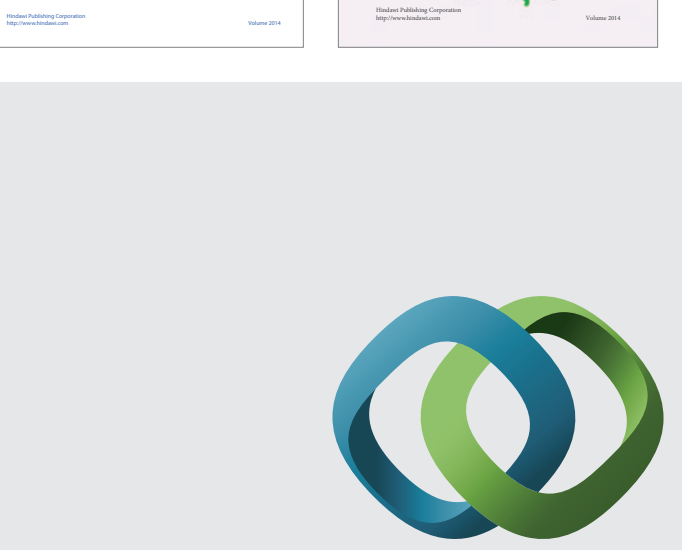

\section{Hindawi}

Submit your manuscripts at

http://www.hindawi.com
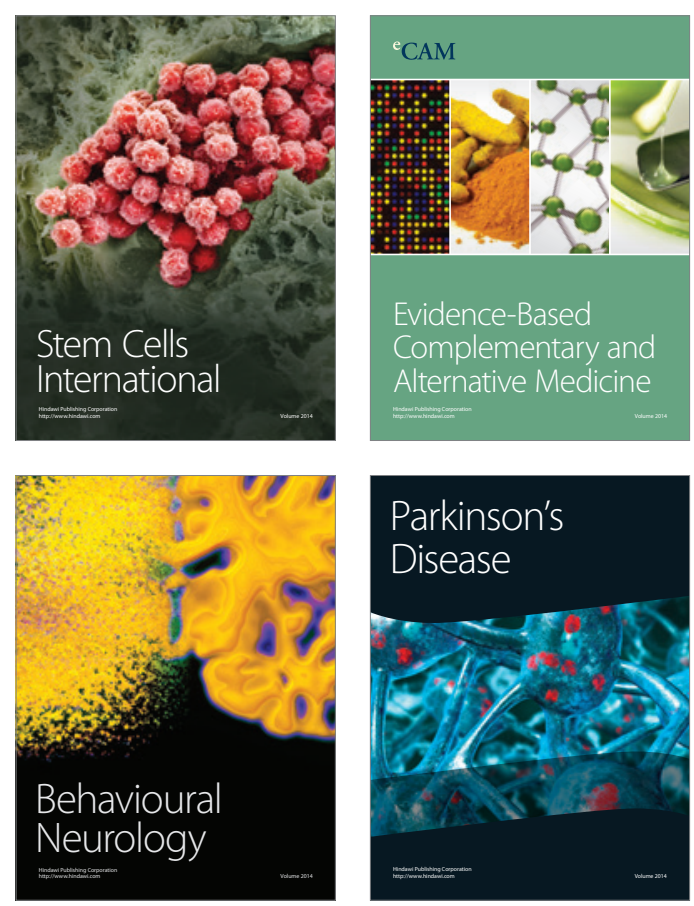

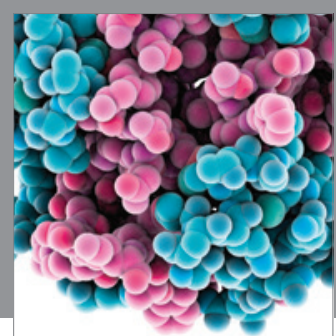

Journal of
Diabetes Research

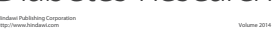

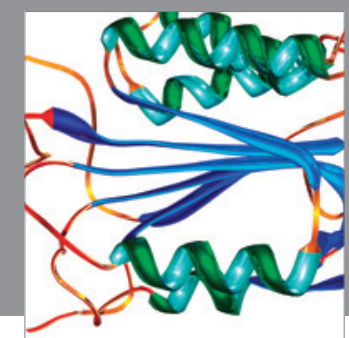

Disease Markers
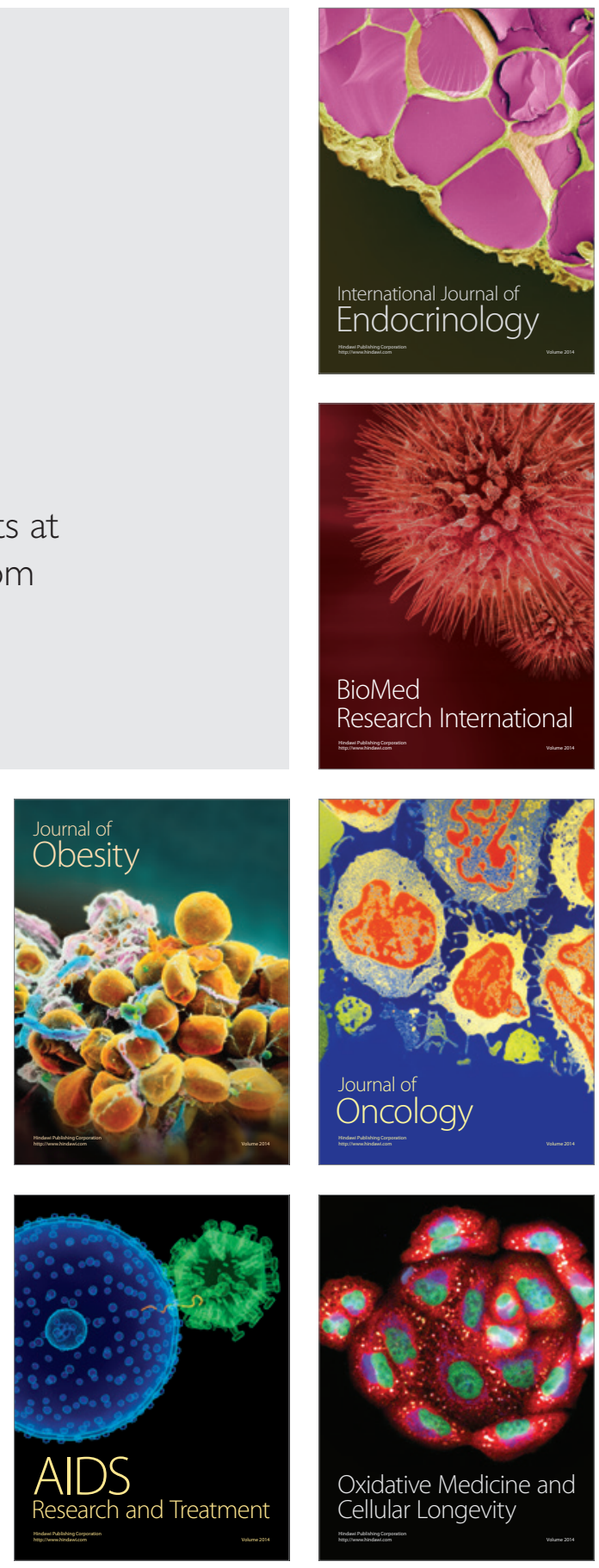